\title{
Medienbildung
}

\section{Korina M. Jocson: Youth Media Matters: Participatory Cultures and Literacies in Education}

Minneapolis: University of Minnesota Press 2018, 186 S., ISBN 9780816691869, USD 25,-

Dass die medialen Produktionen von Jugendlichen wissenschaftliche Wahrnehmung finden, ist nicht selbstverständlich. Korina M. Jocson zeigt in Youth Media Matters verschiedene Lehrsituationen auf, in denen sie die Medienproduktion junger Menschen angeleitet oder forschend begleitet hat. Ziel war stets die Förderung von partizipativer politischer Kultur und Medienkompetenzen - bei Jocson als new media literacies (vgl. S.10) bezeichnet.

Das Buch ist in fünf Kapitel gegliedert, die sie jeweils mit persönlichen Anekdoten einleitet. Die Beschreibung eigener Lehrsituationen sowie die Auswertung ethnografischer Interviews und Feldnotizen nutzt sie zur Konturierung der Konzepte assemblage (Sammlung), critical solidarity (konstruktiver künstlerischer Austausch) und place-making (Mitgestaltung der eigenen lokalen Umgebung, wörtl.: Gestaltung eines Platzes). Diese berühren im Kern die didaktischen Prinzipien der Produktorientierung (Ausrichtung einer Unterrichtseinheit an einem Produkt, z.B. Video), des Lebensweltbezugs (Anbindung unterrichtlicher Inhalte an die reale Umgebung Jugendlicher) sowie die Förderung der Selbstwirksamkeit im Zeitalter neuer medialer Formate.

So erklärt Jocson beispielsweise anhand einer Unterrichtseinheit, in der Schüler_innen eigene Videos über die Wege ihrer Vorfahren nach Amerika erstellten, was assemblage bedeutet: Die entstandenen Kollektionen aus Interviews, eingescannten Bildern, Informationen und musikalischer Untermalung verdeutlichten, wie leicht solche Techniken mittlerweile auch für Ungeschulte umsetzbar sind und Produkte einer Unterrichtseinheit darstellen können. Eng damit verknüpft ist das Konzept der critical solidarity, welches Jocson anhand zweier ,multimedialer Gedichte' erklärt (vgl. S. 45f.). Diese käme durch Interaktion mit digitalen Medien zustande, beispielsweise in Form von kollaborativ erstellten Gedicht-Adaptionen im Videoformat, wodurch neue Möglichkeiten der sozialen und partizipativen Interaktion entstehen. Diese könnten auch über den eigenen Lernkontext hinaus zur kritischen und fruchtbaren Zusammenarbeit mit anderen führen. Solche Interaktion könne auch zur Förderung der new media literacy beitragen und kritische oder politische Auseinandersetzung mit medialen Produkten sowie die Erweiterung der eigenen Handlungs- und Erfahrungshorizonte im Zusammenhang mit neuen Medien ermöglichen (vgl. S.10). Weiterhin wagt Jocson anhand des Videoprojekts einer ,Problemschule‘ eine eigene Definition des Konzepts place-making aufzustellen. Die Jugendlichen erklä- 
ren dabei in selbst produzierten Videos auf der Grundlage von Geodatenanalysen, welche Bedeutung der Ort, an dem sie leben, für sie hat. Place-making wird demnach von der Autorin eher als Reflexion der Lernumgebung, denn als aktive Einflussnahme auf die Gestaltung einer gemeinschaftlichen Umgebung verstanden

Die Projekte sind in einer Detailfülle beschrieben, die es Lehrkräften ermöglicht, sie als Vorlage für eigene Lehrprojekte zu nutzen. Sie profitieren zwar häufig von nicht immer vorauszusetzenden Ressourcen, wie der Verfügbarkeit eines Videostudios, oder einem besonders großen zeitlichen Rahmen, wodurch eine eigene Durchführung zunächst eher schwierig erscheint. Jedoch sind die Unterrichtsformate, die sie präsentiert, grundsätzlich erstrebenswert.

Durch die präsentierten Lehrprojekte werden die Medienprodukte Jugendlicher nicht nur besser wahrgenommen, sondern sie werden im gleichen Zuge zum Unterrichtsziel und tragen zur Steigerung des Gefühls der Selbstwirksamkeit bei. Die Förderung der new media literacy ist ein nobles Ziel, und sie benötigt, wie Jocson selbst fest- stellt, eine neue Haltung („mindset“, vgl. S.22), die etwa das Bewusstsein über die Bedeutung von Privatsphäre im digitalen Raum oder die Selektivität von Hashtags mit einschließt. Die Thematisierung solcher Aspekte wird in den Beschreibungen der Projekte leider nicht erwähnt, weshalb die Konstruktion von Lehrszenarien zur Entwicklung informierter geistiger Haltungen der Lernenden Aufgabe der Leser_innen bleibt.

Sowohl die ausgewählten Themen als auch die enge Verknüpfung unterschiedlicher Materialien lassen dieses Buch jenseits vom antizipierten Format einer wissenschaftlichen Analyse zu einer textuellen assemblage aus Erfahrungsbericht, empirischer ethnografischer Untersuchung und politischer Stellungnahme werden. Es bezieht seine Relevanz nicht aus seinen Bezügen zu zahlreichen philosophischen und erziehungswissenschaftlichen Theorien, die jeweils knapp angerissen werden, sondern aus der Aktualisierung des Nachdenkens über didaktische Prinzipien im Kontext neuer Medientechnologien.

Sophia Hercher (Marburg) 